\title{
Functional Optimization Properties of Median Filtering
}

\author{
Guoping Qiu, Member, IEEE
}

\begin{abstract}
In this letter, we use a new approach for studying the properties of median filtering. Specifically, using threshold decomposition, it is shown that median filtering operation minimizes a two-term cost function of the output state of the median filter. The first term of the cost function measures the smoothness between the median filter output and its neighbor points within the operation window, and the second term measures the discrepancy between the filter output and its original signal. The results from this study have provided us with a new tool to analyze and understand some of the properties of the median filtering operation, including weighted median filtering.
\end{abstract}

\section{MEDiAN FILTERING}

$\mathbf{M}$ EDIAN FILTERING is a discrete time process in which a $2 N+1$ points wide window is stepped across an input signal. At each step, the points inside the window are ranked according to their values, and the median value of the ranked set is taken as the output value of the filter for each window position. Consider a real, discrete-time sequence $\{a(n)\}$, where $a$ is a $M$-level signal. The output of the median filter $y(n)$ is given by $y(n)=$ median $[a(n-N), \ldots, a(n), \ldots, a(n+N)]$. One of the properties of the median operation is that it commutes with a thresholding operation [1]. Thus, multilevel running median can be equivalently obtained by thresholding the input signal at all possible levels, filtering each of the binary thresholded signals by a median filter, and adding these binary outputs to produce the final result [1]. Define $\left\{a_{i}(n)\right\}$, $i=1,2, \ldots, M-1$ as the $i$ th level binary signal sequence of $\{a(n)\}$, where $a_{i}(n)=0$ if $a(n)<i$, and $a_{i}(n)=1$ if $a(n) \geq$ $i$. The output of the median filter at the $i$ th level is given by $y_{i}(n)=$ median $\left[a_{i}(n-N), \ldots, a_{i}(n), \ldots, a_{i}(n+N)\right]$. According to [1], we have

$$
y(n)=\sum_{i=1}^{M-1} y_{i}(n)
$$

\section{MEdian Filtering is an Optimization Process}

It has been shown in [1] that median filtering of an arbitrary level signal is equivalent to decomposing the signal into binary signals, filtering each binary signal with a binary median filter, and then reversing the decomposition. In this section, we analyze the functional minimization property of the binary median filtering operation. For analysis purposes, we transfer

Manuscript received December 7, 1993; approved March 24, 1994. The associate editor coordinating the review of this letter and approving it for publication was Dr. P. Duhamel.

The author is with the School of Computing and Mathematics, University of Derby, Derby, England.

IEEE Log Number 9401806. the $\{0,1\}$ binary of $\left\{a_{i}(n)\right\}$ into $\{-1,1\}$ binary of $\left\{b_{i}(n)\right\}$ by the operation: $b_{i}(n)=2 a_{i}(n)-1$. Define $V_{i}(n)$ as the output of median filter of $\left\{b_{i}(n)\right\}$, which is given by $V_{i}(n)=$ median $\left[b_{i}(n-N), \ldots, b_{i}(n), \ldots, b_{i}(n+N)\right]$, and $V_{i}(n)=2 y_{i}(n)-1$.

For median filtering of the binary sequence $\left\{b_{i}(n)\right\}$, we can write the output of the median filter as follows:

$$
V_{i}(n)= \begin{cases}+1 & \text { if } S(n) \geq 0 \\ -1 & \text { otherwise }\end{cases}
$$

where

$$
S(n)=\sum_{\substack{j=-N \\ j \neq 0}}^{j=N} b_{i}(n+j)+b_{i}(n) .
$$

We define a cost function of the output state of the median filter as follows:

$$
E_{i}\left(V_{i}(n)\right)=-\sum_{\substack{j=-N \\ j \neq 0}}^{j=N} V_{i}(n) b_{i}(n+j)-V_{i}(n) b_{i}(n) .
$$

It is straightforward to show that the median filter operation of (2) always forces (4) into its minimum: $E_{i}(+1)=-S(n)$ and $E_{i}(-1)=S(n)$. If $V_{i}(n)=+1, S(n) \geq 0, E_{i}(+1)<$ $E_{i}(-1)$. If $V_{i}(n)=-1, S(n)<0, E_{i}(-1)<E_{i}(+1)$. We called this property of the median filtering the functional optimization property. In each level of the thresholded space, this functional optimization property holds. Thus, we can state that median filtering is an optimization operation in which the output of the filter is always set to the minimum of a cost function of the output state of the filter. We call the cost function of (4) the median cost function.

Now, we are going to explain the meaning of (4). It may seem strange at first glance that we write the equation into two terms, instead of a single sum. As will become clear in the following analysis, these two terms each has a different meaning. The first term measures the smoothness between the filter output and its neighbor points within the filtering window, and the second term measures the discrepancy between the filter output and the original signal.

If the output of the filter is identical to its input neighbor $\left(V_{i}(n)=b_{i}(n+j), j \neq 0\right)$, the median cost function is reduced, and if the filter output is different from its neighbor $\left(V_{i}(n) \neq b_{i}(n+j), j \neq 0\right)$, the median cost function is increased. Because the median filtering operation (2) always minimizes the cost function, it will encourage the output to have the same value as its neighbors. Therefore, the median filtering has the tendency of smoothing the signal. The second 
term of (4) encourages the filter output to take the same value as its original signal. If the filter output is identical to its original signal $\left(V_{i}(n)=b_{i}(n)\right)$, the value of the cost function is reduced. Conversely, if the filter output differs from its original signal $\left(V_{i}(n) \neq b_{i}(n)\right)$, the cost function is increased. Again, because the median filtering operation (2) always minimizes the median cost function, it will encourage the output of the median filter to take the same value as its original signal.

It is clear from the above analysis that the function of median filtering is a combination of two aspects: The median filtering favors the filtered signal to be smooth, and it encourages the filtered signal to be the same as the original signal. This may explain why median filters has the essential characteristics of removing noise without extensive blurring and edge destruction.

This functional optimization property can be easily extended to analyze other forms of median filtering operations, such as weighted median filter [2], including center-weighted median filters [3].

\section{Center-Weighted Median Filtering}

Center-weighted median filters [3] are a special case of weighted median filters [2] and are given by the median over a modified set of observations containing multiple $k$ center samples. Specifically, center-weighted median are defined by

$$
\begin{gathered}
V_{i}(n)=\operatorname{median}[b_{i}(n-N), \ldots, \underbrace{b_{i}(n) b_{i}(n), \ldots b_{i}(n)}_{k}, \\
\left.b_{i}(n+1), \ldots, b_{i}(n+N)\right] .
\end{gathered}
$$

For center-weighted median filtering of a binary sequence $\left\{b_{i}(n)\right\}$, we can write the output of the median filter as follows:

$$
V_{i}(n)= \begin{cases}+1 & \text { if } S(n) \geq 0 \\ -1 & \text { otherwise }\end{cases}
$$

where

$$
S(n)=\sum_{\substack{j=-N \\ j \neq 0}}^{j=N} b_{i}(n+j)+k b_{i}(n)
$$

The median cost function of the center-weighted median filter is defined as follows:

$$
E_{i}\left(V_{i}(n)\right)=-\sum_{\substack{j=-N \\ j \neq 0}}^{j=N} V_{i}(n) b_{i}(n+j)-k V_{i}(n) b_{i}(n) .
$$

It was shown in [3] that for center-weighted median filters, there exist a tradeoff between detail preservation and noise suppression properties. By observing the median cost function of this filter (see (8)), these properties can be easily understood. It was statistically shown in [3] that center-weighted median filter with a larger center weight (a larger $k$ ) performs better in detail preservation than one with a smaller centre weight (a smaller $k$ ). From (8), this property can be readily understood. For a larger $k$, the discrepancy term in the equation takes more weight, and thus, the median filter output will be more likely to take the value of the original signal than it would be for a smaller value of $k$. For example, if the filter output is different from its original signal $\left(V_{i}(n) \neq b_{i}(n)\right)$, the discrepancy term of the cost function is increased by $k$, the larger $k$ is, the more it is increased, and therefore, the larger $k$ is, the more likely $V_{i}(n) \neq b_{i}(n)$ will not be the minimum of the cost function. Recall that the median filtering operation always minimizes the cost function; therefore, the larger $k$ is, the more likely $V_{i}(n) \neq b_{i}(n)$ will not be the output of the filter. Conversely, if the filter output is identical to its original signal $\left(V_{i}(n)=b_{i}(n)\right)$, the discrepancy term of cost function is decreased by $k$, and the larger $k$ is, the more it is decreased, and the more likely $V_{i}(n)=b_{i}(n)$ would be the minimum of the cost function. Again, due to the functional minimization property of the median operation, the more likely $V_{i}(n)=b_{i}(n)$ would be the output of the filter. It can also be understood from another point of view; for a larger value of $k$, the smooth term in (8) takes a relatively less weight than it would be for a smaller value of $k$, and thus, the filter output will tend to take the value that is less smooth between it and its neighbor points within the operation window than it would be otherwise. Therefore, for larger value of $k$, the noise suppression capability of the center-weighted median filter is reduced while its detail preservation capability is increased.

\section{CONClusion}

In this letter, we have shown that the median filtering operation is an optimization process in which a cost function of the states of the filter output is minimized. The introduction of a cost function associated with median filtering has provided use with a new tool for analyzing and intuitively understanding some of the properties of median filters.

\section{REFERENCES}

[1] J. P. Fitch, E. J. Coyle, and N. C. Gallagher, Jr., "Median filtering by threshold decomposition," IEEE Trans. Acoust. Speech Signal Processing, vol. 32, pp. 1183-1188, 1984.

[2] D. R. K. Brownrigg, "The weighted median filter," Comm. ACM, vol. 27 , pp. 807-818, 1984.

[3] S. J. Ko and Y. H. Lee, "Centre weighted median filters and their applications to image enhancement," IEEE Trans. Circuit and Systems, vol. 38, pp. 984-993, 1991. 